Check for updates

Cite this: RSC Adv., 2017, 7, 22378

\title{
Negative thermal expansion of pure and doped graphene
}

\author{
Sarita Mann, Ranjan Kumar and V. K. Jindal (iD *
}

Graphene and its derivatives distinguish themselves for their large negative thermal expansion even at temperatures as high as $1000 \mathrm{~K}$. The linear thermal expansion coefficients (LTEC) of two-dimensional honeycomb structured pure graphene and B/N doped graphene are analyzed using $a b$ initio density functional perturbation theory (DFPT) employed in VASP software under quasiharmonic approximation. One of the essential ingredients required is the phonon frequencies for a set of points in the Brillouin zone and their volume dependence. These were obtained from the dynamical matrix which was calculated using VASP code in interface with phonopy code. In particular, the transverse acoustic modes (ZA) behave drastically differently as compared to planer modes and so also their volume dependence. Using this approach firstly thermal expansion for pure graphene is calculated. The results agree with earlier calculations using similar approach. Thereafter we have studied the effect of boron and nitrogen doping on LTEC. The LTEC of graphene is found to be negative in the entire range of temperature under study $(0-1000 \mathrm{~K})$ and its value at room temperature (RT) is around $-3.26 \times 10^{-6} \mathrm{~K}^{-1}$. The value of LTEC at RT becomes more negative with B/N doping in graphene. In order to get an insight into the cause of negative thermal expansion, we have computed the contribution of individual phonon modes of vibration. We notice that it is principally the ZA modes which are responsible for negative thermal expansion. It has been concluded that transverse mode in 2D hexagonal lattices have an important role to play in many of the thermodynamical properties of $2 \mathrm{D}$ structures. We have extended the study to calculate the LTEC of $h-B N$ sheet also.

Received 8th February 2017 Accepted 17th April 2017

DOI: $10.1039 / \mathrm{c} 7 \mathrm{ra01591g}$

rsc.li/rsc-advances

\section{Introduction}

The first successful experimental synthesis of graphene, ${ }^{1}$ a $2 \mathrm{D}$ thermodynamically stable structure of carbon, created a great deal of research interest. In graphene, $\mathrm{sp}^{2}$ hybridized carbon atoms are arranged in a honeycomb lattice structure. It has attracted great scientific interest mainly because of its unusual electronic properties. In fact it has been identified as a very promising candidate as a future application material, based on its chemical and mechanical stability along with ballistic transport at room temperature (RT). ${ }^{2}$ Its charge carriers exhibit very high intrinsic mobility. Its special electronic transport which is governed by a Dirac-like-equation makes it act as a bridge between the condensed matter physics and electrodynamics. ${ }^{3-5}$ There has been a lot of research ongoing to study and modify electronic and optical properties of graphene by doping with a variety of atoms. We in our previous research ${ }^{6-8}$ have studied the band gap modification and optical behavior by optical behavior by replacing some percentage of the carbon atoms with nitrogen/boron atom dopants.

Department of Physics, Panjab University, Chandigarh-160014, India. E-mail:Jindal@ pu.ac.in
Equally important aspect of graphene is its unique thermal properties. Its thermal conductivity is unusually high ${ }^{9}$ which is a subject of intensive research. Heat flow hindrance in miniature components is a matter of concern in the micro and nanoelectronics. There is a lot of research ongoing in this area. In addition to this, graphene also has unusual negative linear thermal expansion coefficient (LTEC) as reported by several authors. ${ }^{10-15}$ Mounet et al. ${ }^{10}$ have made a detailed study of LTEC of carbon materials including graphene using first-principles based quantum espresso code under quasi harmonic approximation (QHA). They report the LTEC for graphene upto temperatures as high as $2500 \mathrm{~K}$ with a RT value around $-3.6 \times$ $10^{-6} \mathrm{~K}^{-1}$. Calculations made by Monte Carlo simulations ${ }^{11}$ estimated the average value of LTEC of graphene to be $-4.8 \times$ $10^{-6} \mathrm{~K}^{-1}$ between the temperatures ranges $0-300 \mathrm{~K}$ and estimated negative behavior only up to $900 \mathrm{~K}$. We also notice a calculation ${ }^{12}$ determining the LTEC of graphene using nonequilibrium green's function with and without substrate. They report large negative values of LTEC at low temperature region and small positive values at high-temperature region, with a RT value of $-6 \times 10^{-6} \mathrm{~K}^{-1}$. Thereafter Sevik ${ }^{13}$ has reported similar results as ${ }^{10}$ for graphene using DFPT implemented in VASP. However none of the above theoretical calculations bring out clearly the origin of the negative thermal expansion though it 
remains a feature of $2 \mathrm{D}$ systems which is also found to be reported by Kim et al. ${ }^{\mathbf{1 4}}$ who observe it for graphene too. Recently, Jiang et al. ${ }^{15}$ have reviewed the significance of flexural mode of graphene. They report that flexural mode is responsible for high negative contribution to thermal expansion of graphene by studying the contribution of individual phonon modes to thermal expansion. Michel et al. ${ }^{\mathbf{1 6}}$ have made a detailed analysis on the role of anharmonic phonons on 2D crystals reporting negative thermal expansion whenever flexural modes are involved. They ${ }^{\mathbf{1 7}}$ also report selective contribution from various acoustic modes to thermal expansion in graphene and introduce finite size to check the divergent Grüneisen parameter from flexural modes.

Experimental studies of thermal expansion of graphene have also been reported. ${ }^{18-20}$ Bao et al. ${ }^{18}$ have measured the change in length of suspended graphene sample and estimated the LTEC of graphene in a narrow range of temperature (300-400 K), which remains negative only upto $350 \mathrm{~K}$ with a RT value of $-7 \times$ $10^{-6} \mathrm{~K}^{-1}$. Yoon et al. ${ }^{19}$ have measured temperature dependent Raman shift in $\mathrm{G}$ band of graphene and analytically estimated the LTEC in temperature range of (200-400) K, with RT value of $(-8 \pm 0.7) \times 10^{-6} \mathrm{~K}^{-1}$. Similarly, Pan W. et al. ${ }^{20}$ also report useful data for thermal expansion in the high temperature range from RT to above $1000 \mathrm{~K}$ from the measured Raman shifts of $\mathrm{G}$ and 2D peaks in graphene. Most of the theoretical estimates ${ }^{\mathbf{1 0 - 1 7}}$ underestimate the experimental observations.

The h-BN (hexagonal-boron nitride) sheet is another twodimensional (2D) material which has attracted great attention in last few years due to its interesting application related properties. $^{21,22}$ The h-BN crystal structure is similar to graphene as boron and nitrogen atoms are arranged in $\mathrm{sp}^{2}$ bonded $2 \mathrm{D}$ hexagonal structure. In the h-BN sheet, nitrogen and boron atoms are attached by strong covalent bonds. Despite the polar nature of the $\mathrm{BN}$ bond in contrast to graphene which lead to significant electronic structural changes, its elastic properties remain similar. h-BN distinguishes itself also because of a large band gap of 5-6 eV as compared to zero band gap in graphene. ${ }^{23}$ It also shows high thermal stability, large dielectric breakdown and enhanced chemical inertness compared to graphene. On account of these properties, it becomes a useful material for applications in industries. Therefore, it follows naturally that the unique electronic properties are supplemented by a study of their thermodynamic and phonon related properties as well, only then the full potential in the applications of graphene or doped graphene or BN sheet is exploited. Sevik ${ }^{\mathbf{1 3}}$ has also studied LTEC of h-BN sheet using VASP software and reported negative thermal expansion with almost double the RT value as compared to graphene. Thomas et al. ${ }^{24}$ have studied the LTEC of h-BN sheet using molecular dynamics and shows negative LTEC at low temperature.

Negative LTEC is crucial, as it is reported in almost all 2D substances. Moreover, this phenomenon initiates at low temperature. At finite temperature, LTEC is negative due to outof-plane vibrations as reported in theoretical calculation ${ }^{25}$ of self-consistent phonons.

We have previously studied the phonon dispersion and various thermal properties like specific heat, entropy and free energy of pure graphene and how they varied with different $\mathrm{B}$ and $\mathrm{N}$ doping concentrations for graphene ${ }^{26}$ where we also reported some estimates of LTEC for pure graphene. Since these estimates gave us negative values of thermal expansion, we now recalculate the estimates by carefully understanding the origin of negative expansion.

Therefore apart from study of thermal expansion, the primary goal of this paper has been to emphasize on the reasons for negative thermal expansion by focusing on contribution by individual phonon branches. Due to the 2-D structure of graphene with 2 atoms per unit cell, the 4 external phonon branches are usually present with 2 acoustic and 2 optic ones. The external branches arise due to intermolecular vibrations representing lattice modes. We calculate their contribution to thermal expansion separately. The other two branches in the transverse direction identified as ZA (transverse acoustic) and $\mathrm{ZO}$ (transverse optic) originate due to quasi-2D behavior and are very special modes of vibration for $2-\mathrm{D}$ structures. Their restoring forces are derived from in plane modes and these behave very differently. Realizing that the Grüneisen parameter for ZA mode is largely negative, we have focused on its contribution separately from other branches and found that it is the most important branch which accounts for NTE in graphene. Although a detailed study for pure graphene looking into the negative thermal expansion has been made $^{17}$ but we have extended the LTEC study of graphene to doped graphene also by varying the boron and nitrogen dopant concentrations leading to h-BN sheet. Therefore despite the fact that quite a lot has been studied recently outlining the significance of ZA mode on thermal properties of graphene, this study supplements the recent study on the subject, giving detailed results of temperature dependence on thermal expansion and comparing most of the available results. Since pure graphene is not a suitable material for device applications, a study of doped graphene based on $\mathrm{B}$ or $\mathrm{N}$ will be of great practical use.

The present paper is structured as follows. After Introduction in Section 1 where graphene, the overview of its various electronic and thermodynamic properties and h-BN and its various properties are presented, a brief summary of theory and computational details are presented in Sec. 2. Section 3 gives results and discussion which includes lattice thermo-dynamical properties like thermal expansion, surface bulk modulus and mode Grüneisen parameters as acquired from the variation of free energy with volume for pure graphene, $\mathrm{B}$ and $\mathrm{N}$ doped graphene and h-BN sheet. Sec. 4 contains Summary and conclusions.

\section{Theory and computational details}

\subsection{Theory}

To calculate the frequencies of phonon, the VASP (Vienna Abinitio Simulation Package $)^{27,28}$ code which is a density functional theory (DFT) based software was used in combination with phonopy ${ }^{29}$ software. The VASP software is based on $a b$ initio density functional theory. The position coordinates of the atoms in unit cell and lattice parameters are given as input to the software with necessary conditions set by input parameters 
for cell relaxation. After relaxing the cell for minimum energy configuration, the lattice parameter of the structure is calculated. The structure is given small perturbations through displacements of atoms and VASP is again run under density perturbation theory for calculation of the dynamical matrix. The phonopy code uses the dynamical matrix obtained in the VASP to calculate the phonons and related thermodynamic properties. Under quasi harmonic approximation, thermal expansion gives rise to implicit shift in phonon frequencies and can be procured from dependence of the phonon frequencies on volumes, termed as Grüneisen parameter that can be defined $^{30,31}$ as

$$
\gamma_{\mathbf{q} j}=-\frac{\partial \ln \omega}{\partial \ln V}=-\frac{V_{0}}{\omega_{\mathbf{q} j}} \frac{\partial \omega_{\mathbf{q} j}}{\partial V}
$$

where $V_{0}$ is equilibrium volume, $\mathbf{q}$ is the phonon wave vector, $j$ stands for different phonon branches and $\omega_{\mathbf{q} j}$ 's the harmonic phonon frequencies at particular values of $\mathbf{q}$ and $j$.

The mode Grüneisen parameters are the key ingredient in thermal expansion mechanisms. These parameters are generally positive but for some modes and for specific q values, they are found to be negative in a narrow temperature range. They are highly negative for lowest acoustic mode in case of graphene which gives negative values for thermal expansion coefficient at low temperatures. This may be due to that fact that only lower acoustic modes can be excited at low temperatures.

In absence of external pressure, the equilibrium structure of a crystal structure can be obtained at temperature $T$ by free energy minimization w.r.t. its degrees of freedom.

The expression for quasi-harmonic free energy is written as ${ }^{30}$

$$
F_{\mathbf{q} h}=\phi(V)+k_{\mathrm{B}} T \sum_{\mathbf{q}, j} \ln \left[2 \sinh \left(\frac{\hbar \omega_{\mathbf{q} j}}{2 k_{\mathrm{B}} T}\right)\right]
$$

where $\phi(V)$ is the static lattice contribution which can be written $^{30}$ as

$$
\phi(V)=\phi\left(V_{0}\right)+\frac{1}{2} \varepsilon^{2} V_{0}^{2}\left(\frac{\partial^{2} \phi}{\partial V^{2}}\right)=\phi\left(V_{0}\right)+\frac{1}{2} \varepsilon^{2} V_{0} B
$$

where the bulk modulus $B$ is

$$
B=V_{0}\left(\frac{\partial^{2} \phi}{\partial V^{2}}\right)
$$

In case of $2 \mathrm{D}$ structure, we define surface bulk modulus as

$$
B_{\mathrm{S}}=S\left(\frac{\partial^{2} \phi}{\partial S^{2}}\right)=L B
$$

where $S$ is the surface area and $L$ is an arbitrary length which needs to be introduced in the otherwise 3-D standard software.

Using condition for minimum energy, expression for thermal expansion coefficient $\beta$ [derived in ref. 30] is

$$
\beta=\frac{k}{V_{0} B} \sum_{\mathbf{q} j} \gamma_{\mathbf{q} j}\left(\frac{\hbar \omega_{\mathbf{q} j}}{k_{\mathrm{B}} T}\right)^{2} \frac{\mathrm{e}^{\hbar \omega_{\mathbf{q} j} / k T}}{\left(\mathrm{e}^{\hbar \omega_{\mathbf{q} j} / k T}-1\right)^{2}}
$$

LTEC $(\alpha)$ (for 2D materials) is defined as

$$
\alpha(T)=\frac{1}{a(T)} \frac{\mathrm{d} a(T)}{\mathrm{d}(T)}=\frac{1}{2 V} \frac{\partial V}{\partial T}=\frac{1}{2} \beta
$$

As is evident from eqn (6), the thermal expansion involves summation over all branches and the mode dependent Grüneisen parameters. It has been found that mode dependent Grüneisen parameter is very sensitive to the choice of branch. Therefore we introduce $\beta_{j}$ for each branch to calculate branch dependent thermal expansion such that

$$
\beta=\sum_{j} \beta_{j}
$$

\subsection{Computational parameters}

In the calculations, we have made use of the Perdew-BurkeErnzerhof $(\mathrm{PBE})^{32}$ functional which is an exchange correlational functional of generalized gradient approximation (GGA). The cut off energy for plane waves was taken as $750 \mathrm{eV}$. A $4 \times 4$ supercell has been employed which makes a sheet of 32 atoms to simulate the sheet of graphene. Two sheets of graphene are maintained at a separation larger than $10 \AA$ in the transverse direction in order to avoid interlayer interactions. For sampling of Brillouin zone, the Monkhorst-pack scheme is employed. A $\Gamma$ centered $k$ mesh of size $7 \times 7 \times 1$ is used for relaxing the structure. We have made use of tetrahedron method for treating the partial occupancies with Blöchl corrections. ${ }^{33}$ The geometry of coordinates of the system was optimized till the Hellmann-Feynman forces were as low as $0.005 \mathrm{eV}^{-1}$. The phonon frequencies and surface bulk modulus are used in calculation of LTEC using eqn (6) and (7).

\section{Results and discussion}

\subsection{Pure graphene}

The study of pure graphene under quasiharmonic approximation gives an approximate value of Grüneisen parameters and bulk modulus which contribute in calculation of thermal expansion coefficient. We have earlier obtained the phonon

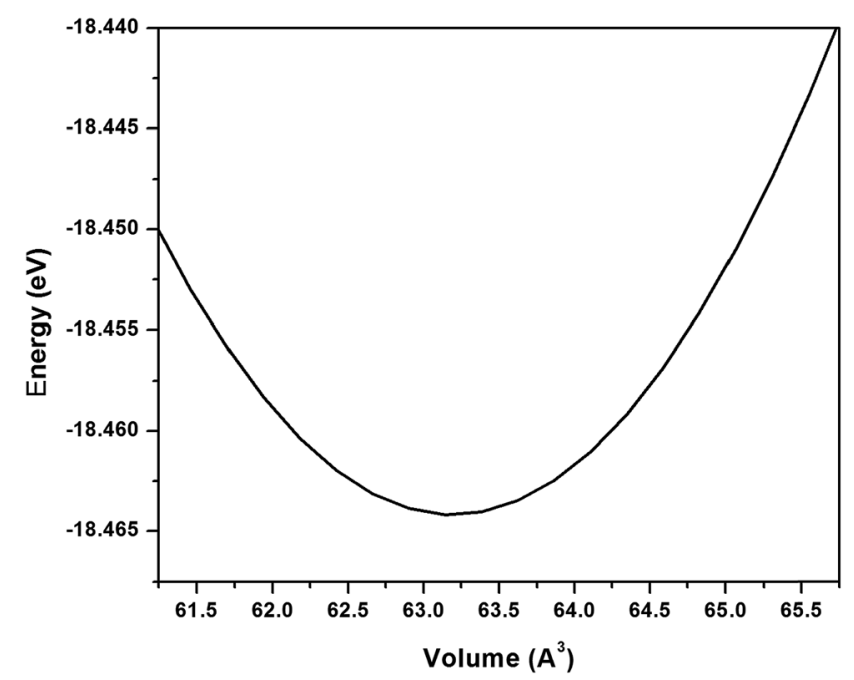

Fig. 1 Energy-volume curve for pure graphene where volume is area multiplied by a constant height in $z$-direction. 
dispersion for pure and $\mathrm{B}$ and $\mathrm{N}$ doped graphene and compared the dispersion curves with various theoretical and experimental results. ${ }^{26}$

3.1.1 Energy-volume curve. Under quasi harmonic approximation, we study the free energy of the structure at different unit cell volumes and found the $E-V$ curve which is shown in Fig. 1. It is to be noted that the volume here is representative of unit cell area. This first derivative of the energy-volume curve gives the minimum unit cell volume of $63.25 \AA^{3}$. The lattice constant corresponding to unit cell of this volume of graphene sheet is $2.47 \AA$. The minimum free energy is $-18.47 \mathrm{eV}$. This value of lattice constant for graphene is very close to $2.46 \AA$ as reported in literature. ${ }^{19}$

Thus bulk modulus using eqn (4) is obtained from second derivative of the fitted curve to the calculated $E-V$ data as shown in Fig. 1. Hence we obtained surface bulk modulus as defined by us (eqn (5)) for pure graphene to be equal to $90.24 \mathrm{~N} \mathrm{~m}^{-1}$. The bulk modulus of graphene has earlier been studied by many authors. ${ }^{34-37}$ Reich et al. ${ }^{34}$ have reported a bulk modulus value of $700 \mathrm{GPa}$ and Milowska et al. ${ }^{37}$ has reported a value of $528 \mathrm{GPa}$ for pure graphene. It may be noted that for calculation of volume of the unit cell they take thickness of the cell around 3.4 A. The $2 \mathrm{D}$ bulk modulus as defined by Kalosakas et $a l^{35}$ has been reported to be $200 \mathrm{~N} \mathrm{~m}^{-1}$ for graphene. We have found a bulk modulus value of 262 GPa which is in qualitative agreement when compared to previous studies based on similar cell volume calculations.

3.1.2 Mode dependent Grüneisen parameter. The Grüneisen parameter plays an important role in finding the dependence of various thermodynamic properties of materials on temperature ranging from phonon frequency shift to thermal expansion of a material. Generally these parameters are positive, as phonon frequencies diminishes when a solid expands, although some Grüneisen parameters are reported to be negative for low frequency acoustic modes and usually combine with positive values from different modes in the end diluting the effectiveness of positive Grüneisen parameters. Interestingly, thermal expansion can not only be diluted by these but also turn positive expansion to a negative expansion. To analyze thermal contraction, we have shown in Fig. 2, mode dependent Grüneisen parameters of graphene. These are obtained from phonon frequencies by interpolation with a polynomial and calculated for the ground state configuration. Interestingly some bands in graphene (lowest transverse acoustic mode) show large negative Grüneisen parameters, as compared with other branches. Thus at very low temperatures when optical modes are still not excited whose Grüneisen are positive, the contribution from the negative Grüneisen parameters will highly contribute to negative thermal expansion. The Grüneisen parameters corresponding to the lowest transversal acoustic ZA modes are negative. These negative Grüneisen parameters will correspond to negative thermal expansion in graphene.

3.1.3 Thermal expansion coefficient. An important feature of thermal properties of graphene is their extremely high thermal conductivity, which makes it a suitable candidate for use as a heat control device in high speed integrated circuits.

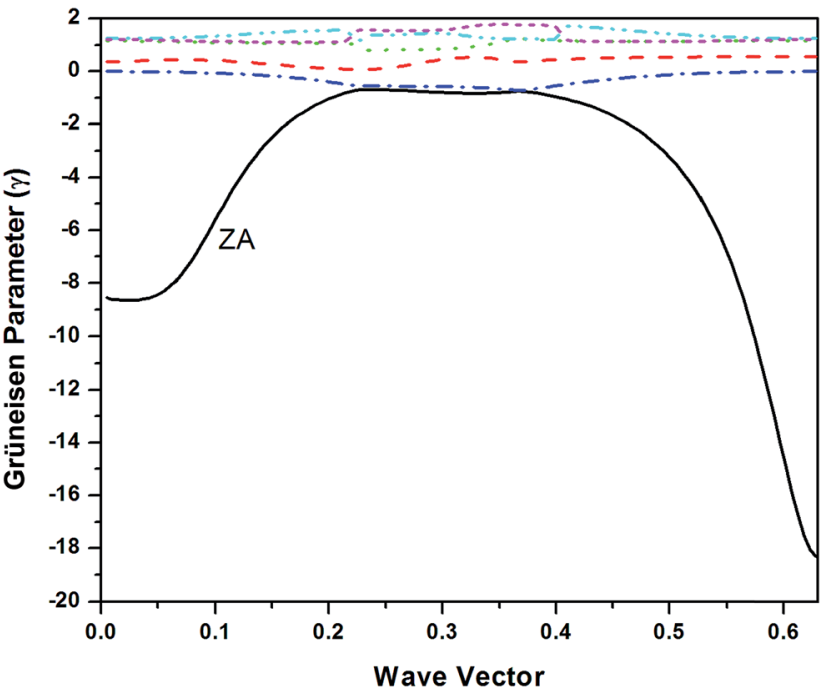

Fig. 2 Grüneisen parameters for various phonon branches of graphene.

Exact knowledge of the temperature dependence of linear thermal expansion coefficient (LTEC) is also important for such applications. Many authors have contributed to the calculation of TEC using various theoretical models $\mathbf{s}^{\mathbf{1 0 - 1 7}}$ and experimental techniques. ${ }^{18-20}$ Bao et al. ${ }^{18}$ have measured experimentally the coefficient of thermal expansion in a very narrow range of temperature (300-400 K) by examining the variation in the sagging of a suspended graphene piece and found it to be negative only up to $\sim 350 \mathrm{~K}$ with a RT value of $-7 \times 10^{-6} \mathrm{~K}^{-1}$. Mounet et al. ${ }^{10}$ estimated the LTEC of graphene using ab initio DFT calculation and estimated that the LTEC of graphene is negative in the whole temperature range under study (0-2500 K). Michel et $a .^{17}$ have studied thermal expansion using anharmonic force constants and estimated the RT value of LTEC to be $-1.6 \times 10^{-7} \mathrm{~K}^{-1}$ which is very much off the experimental values. ${ }^{\mathbf{1 8 - 2 0}}$

As observed in Fig. 3(a), there is a large variation between theoretical and experimental data. Even the experimental data shows variation. In the low temperature regime, the size dependence might have a role to play $^{17}$ - smaller size tends to reduce the large negative value but it has not been mentioned by experimentalists. Our results are in overall agreement with those of Mounet et al. ${ }^{10}$ and show a better agreement with experiments above room temperature.

To get complete and accurate understanding of the behavior of LTEC near room temperature, which is important in designing graphene-based heat devices, we studied the branch dependent LTEC in detail.

DFPT calculations of pure graphene for LTEC calculations show a negative behavior in whole range of temperature under study with a RT value of $\left(-3.26 \times 10^{-6}\right)$. In this calculation we have made use of surface bulk modulus calculated earlier using $E-V$ curve. Our results matches well with the theoretical studies $^{\mathbf{1 0 , 1 3}}$ as shown in Fig. 3(a) but there is still a discrepancy as compared to experimental results. Theoretical 


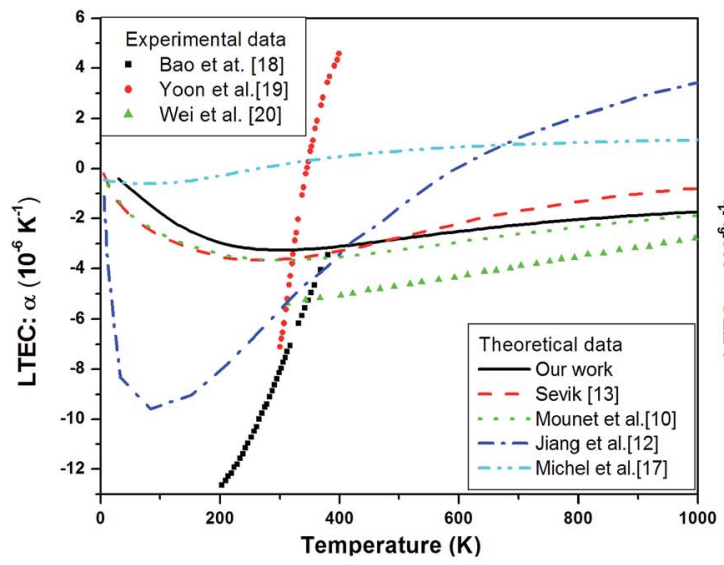

(a)

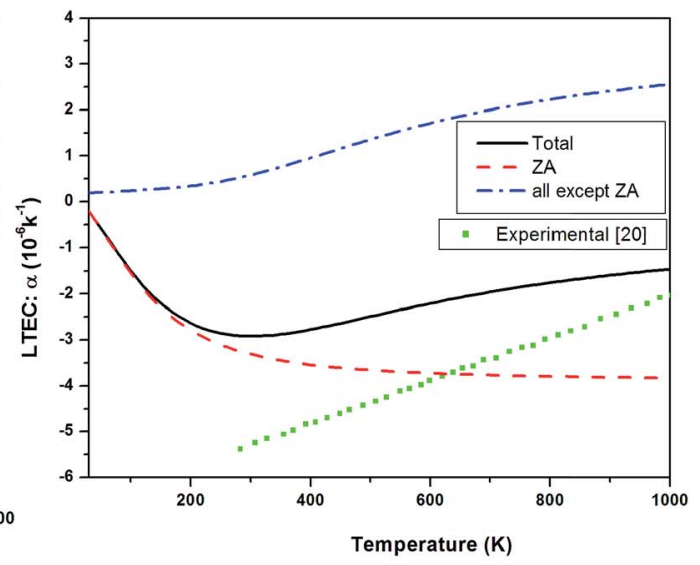

(b)

Fig. 3 (a) Theoretical linear thermal expansion coefficient $\alpha$ of single layer graphene plotted as a function of temperature and compared with experimental data ${ }^{18}$ (black squares), ${ }_{1}^{19}$ (red circles) $a^{2} d^{20}$ (green triangles) and theoretical measurements ${ }^{10}$ (green dots), ${ }_{,}^{13}$ (red dash line), ${ }_{1}^{12}$ (blue dash dot line) and ${ }^{17}$ (cyan dash dot dot line). (b) Branch dependent thermal expansion coefficient (total-black solid line, ZA mode contribution red dash line and all except ZA contribution - blue dash dot line) compared with experimental data ${ }^{20}$ shown by green squares in the curve.

measurements by ref. 12 are very close and ref. 17 are very much off to experimental value of LTEC. The reasons for this disagreement have been attributed to finite size effect incorporated by ref. 17 . To analyze it further, we present partial contributions to LTEC arising from each branch separately as the phonon branch $\mathrm{ZA}$ in particular has large negative Grüneisen parameters and must be visualized separately. Fig. 3(b) shows the branch dependent LTEC which clearly chows that thermal expansion of graphene is negative only due to presence of large negative Grüneisen parameters corresponding to ZA branch. Here we have made use of eqn (8) to calculate branch dependent thermal expansion. The dominant contribution to thermal expansion in graphene is from ZA branch which is negative. The overall thermal expansion is negative due to dominant effect of ZA branch compared to other branches. The experimental data reported here is from three sources ${ }^{18-20}$ all obtaining it from the shift in 2D peak and exploiting thermal expansion as responsible for the shift. However in the small domain of overlap of three measurements i.e. around 300-400 $\mathrm{K}$, there is no agreement between the three. In the high temperature limit above RT our results show a reasonably good agreement with the experimental data. It may be possible that Grüneisen parameter depends on temperature but there is no theoretical or experimental estimate of dependence of Grüneisen parameters on temperature. This assumption introduces uncertainty in estimation of thermal expansion.

\subsection{Doped graphene}

Since the B (N) doped structures are stable upto $25 \%$ doping, ${ }^{26}$ we have done the quasiharmonic study for two cases only i.e. $12.5 \%$ and $25 \%$ The atomic configurations corresponding to doped sheet is shown in Fig. 4.

We have also considered the minimum doping configuration. The minimum doping can be one atom in a sheet of 32 atoms i.e. $3.125 \%$ in this case. But the configuration with low doping i.e. $1 / 2 / 3$ atoms in a sheet is also thermodynamically unstable where almost all of the ZA mode frequencies are imaginary for boron doping and half of ZA frequencies are imaginary for nitrogen doping, as doping of B atoms introduces more lattice mismatch compared to $\mathrm{N}$ atoms doping which makes the structure thermodynamically more unstable. Thus considering one, two or three atoms doping in a hexagonal ring gives the thermodynamically unstable structures. We finally studied thermal expansion in only above two thermodynamically stable structures as already reported in our previous studies. ${ }^{26}$

3.2.1 N-Doping. Thermal expansion coefficient has been calculated for the doped structure for different configurations and compared with that of pure graphene as shown in Fig. 5. There is lowering of LTEC value with increasing doping concentration with a RT value of $-8.19 \times 10^{-6}$ for $12.5 \%$ doping and $-9.66 \times 10^{-6}$ for $25 \%$ doping. The large increase in negative value of thermal expansion with doping is primarily concerned with the ZA branch (similar to that shown earlier for pure graphene in Fig. 3(b)) which has more negative Grüneisen parameters near $\Gamma$ point as low as -150 and -200 for $12.5 \%$ and $25 \% \mathrm{~N}$ doped sheets respectively. The surface bulk modulus values of doped sheets are also depicted using $E-V$ curve to be $106.32 \mathrm{~N} \mathrm{~m}^{-1}$ and $97.2 \mathrm{~N} \mathrm{~m}^{-1}$ for $12.5 \%$ and $25 \% \mathrm{~N}$ doped sheets respectively. It has been shown in an earlier research paper that bulk modulus value of $\mathrm{N}$ doped graphene increases

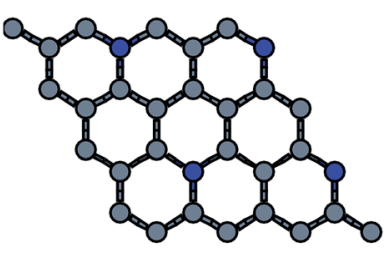

(a)

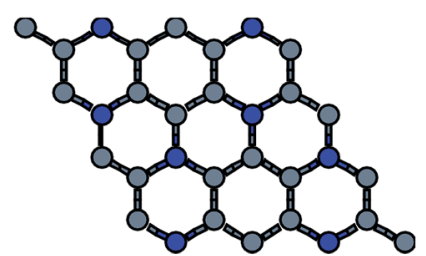

(b)
Fig. 4 Schematic diagrams of (a) $12.5 \%$ and (b) $25 \%$ doping concentrations. 


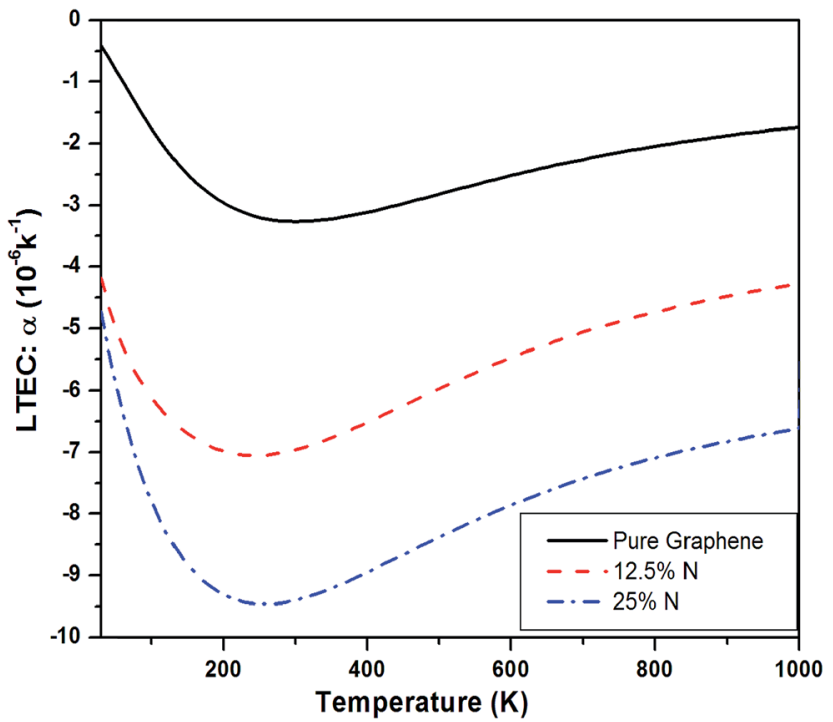

Fig. 5 LTEC of $12.5 \%$ and $25 \% \mathrm{~N}$-doped sheet compared with pure graphene case.

initially with doping and then tend to decrease as doping is increased further. ${ }^{37}$ This is apparently due to decreased cell volume on doping. At higher doping concentrations the modified interactions also play a role.

3.2.2 B-Doping. Thermal expansion coefficient for the B doped structure for different doping configurations is shown in Fig. 6. There is lowering of thermal expansion value with doping with a RT value of $-9.17 \times 10^{-6}$ for $12.5 \%$ doping and $-11.16 \times$ $10^{-6}$ for $25 \%$ doping. The increase in negative value of thermal expansion with $\mathrm{B}$ doping is comparatively larger than $\mathrm{N}$ doping although the trend is similar in both cases. The contribution of $\mathrm{ZA}$ branch in thermal expansion is to make it negative as the Grüneisen parameters for ZA branch for the B doped structures

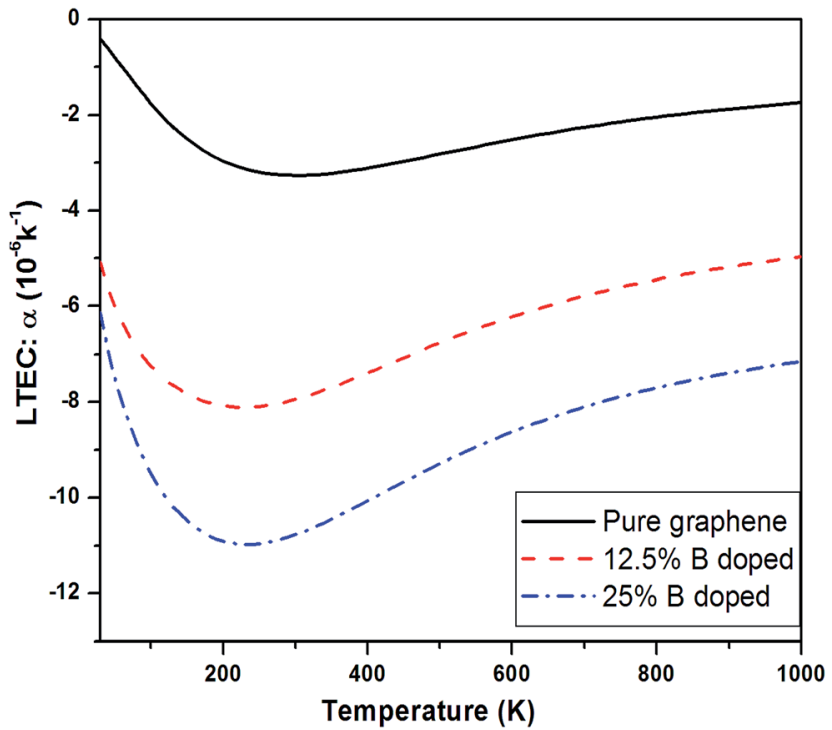

Fig. 6 LTEC of $12.5 \%$ and $25 \%$ B-doped sheet compared with pure graphene case.

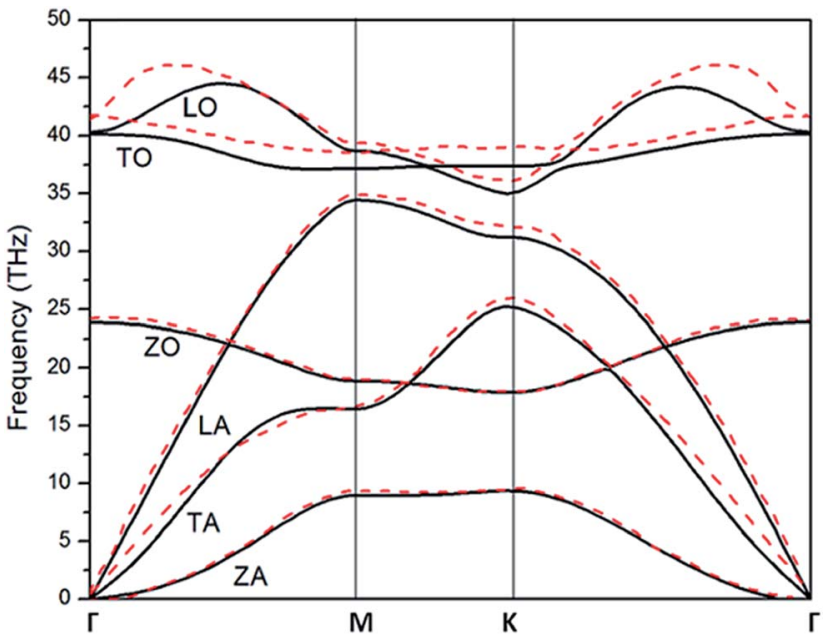

Fig. 7 Phonon dispersion for h-BN (solid lines) compared to that in ref. 38 (red dashed lines). The longitudinal, transversal and out-of-plane acoustical/optical modes are represented by LA/LO, TA/TO and ZA/ZO respectively.

are as low as -105 and -150 for $12.5 \%$ and $25 \%$ respectively. However the increase in negative value of LTEC for the case of B doped sheet as compared to $\mathrm{N}$ doped sheet is much larger due to decrease in its surface bulk modulus value. The surface bulk modulus values of B doped sheets are depicted to be $92.5 \mathrm{~N} \mathrm{~m}^{-1}$ and $73.5 \mathrm{~N} \mathrm{~m}^{-1}$ for $12.5 \%$ and $25 \%$ doping configurations respectively.

\section{3 h-BN sheet}

To understand thermodynamic properties of h-BN sheet, the first step is to inspect lattice vibrational modes (phonons).

3.3.1 Phonon dispersion. Similar to phonon dispersion of graphene calculated earlier, ${ }^{26} \mathrm{~h}$-BN sheet also have 6 phonon branches (three acoustic (A) and $3 \mathrm{~N}-3$ optical (O) phonon modes).

The phonon frequencies are obtained under harmonic approximation which gives phonon dispersion curve of h-BN

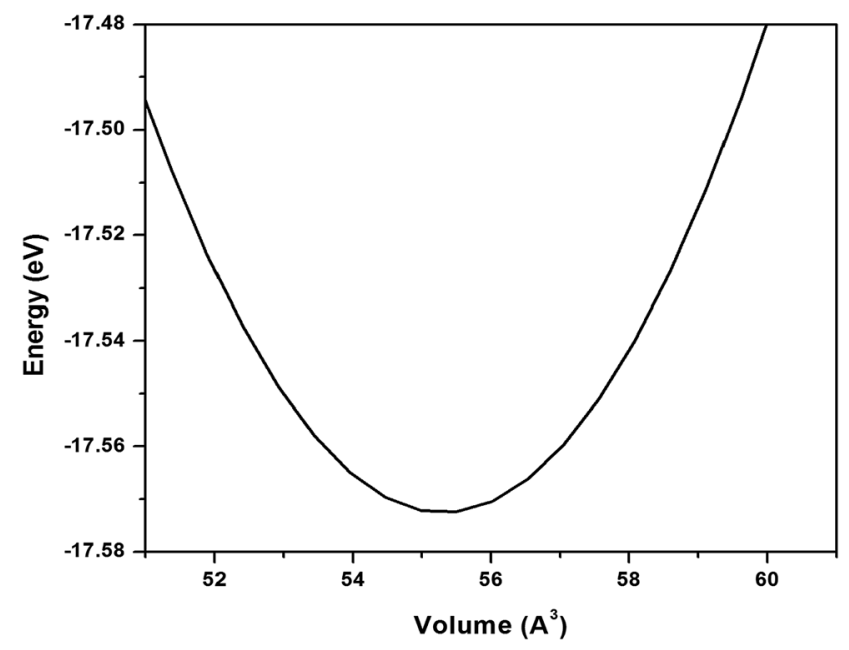

Fig. 8 Energy-volume curve for $\mathrm{h}-\mathrm{BN}$ sheet. 


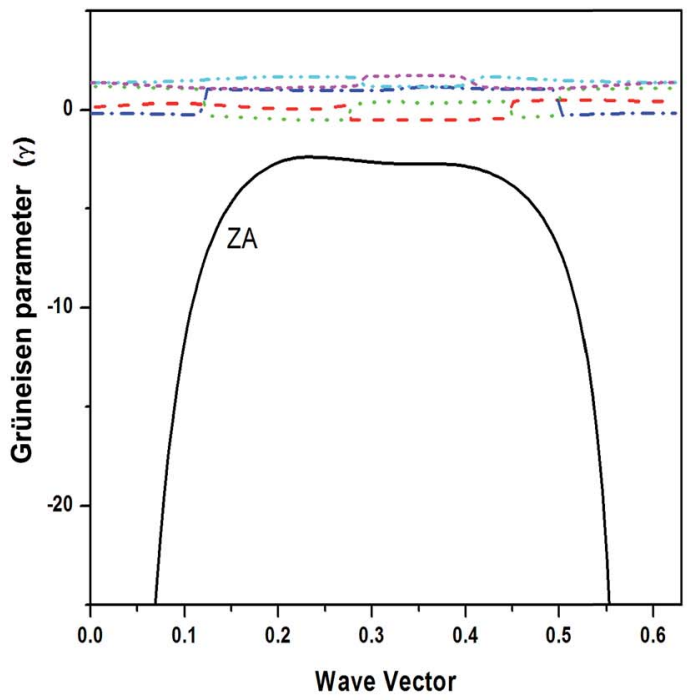

(a)

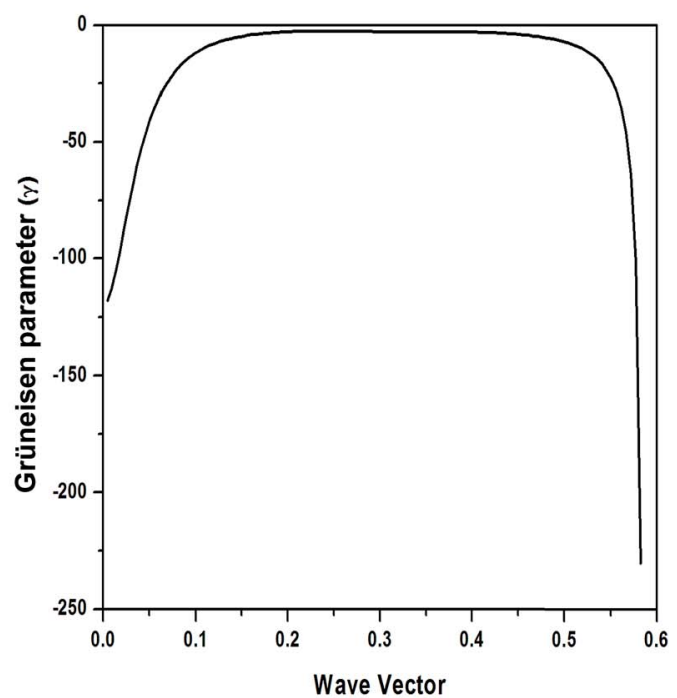

(b)

Fig. 9 (a) Mode dependent Grüneisen parameters, (b) Grüneisen parameter of ZA mode.
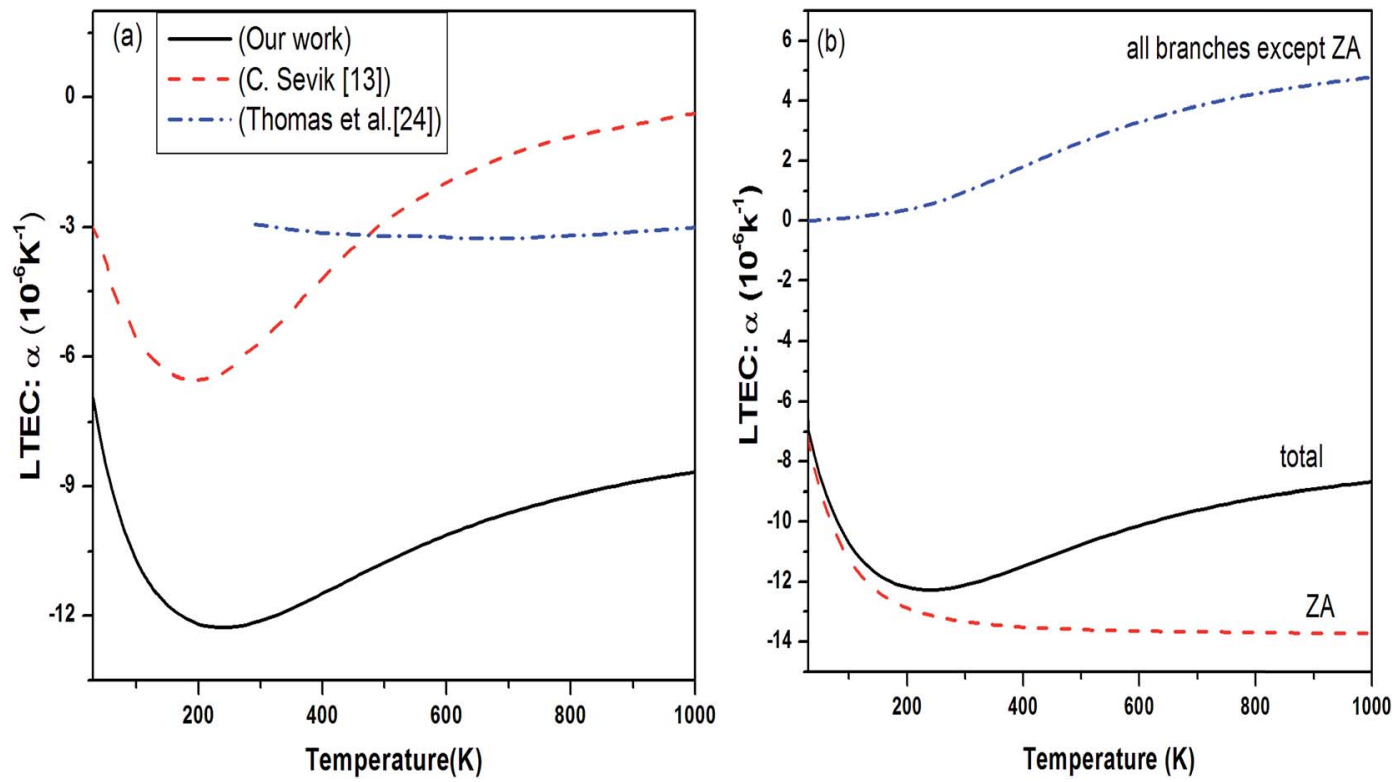

Fig. 10 (a) Temperature dependence of LTEC for single layer h-BN black solid line. Our results are compared with various theoretical measurement (ref. 13 red dashed line and ref. 24 blue dash dot line). (b) Branch dependent thermal expansion.

sheet as shown in Fig. 7. The curves have been obtained by simulating the h-BN sheet over a set of points on the line for different directions between $\Gamma$ points. We have made use of density functional perturbation theory with displacement along all the three directions which give rise to 6 phonon branches in dispersion curve. The phonon dispersion curve is in good agreement with ref. 38 where Wirtz et al. have studied the phonon dispersion curve using molecular dynamics. Our dispersion curve also matches with ${ }^{39}$ where vibrational properties of h-BN are studied. They have shown that in a strictly 2D material, the electrostatic interactions do not produce any macroscopic term at $\Gamma$ point and hence no splitting is observed between LO/TO modes in an h-BN sheet. Our phonon dispersion curve is also fairly in agreement with experimental studies ${ }^{40,41}$ done using inelastic X-ray scattering and Raman analysis methods.

3.3.2 Energy volume curve. Similar to the pure graphene, the $E-V$ curve obtained for h-BN sheet is shown in Fig. 8. The volume (area of sheet multiplied by a constant height along $z$ axis) which minimizes the curve is $55.26 \AA^{3}$.

The lattice constant corresponding to minimum unit cell volume is $2.515 \AA$ A. The surface bulk modulus found to be 


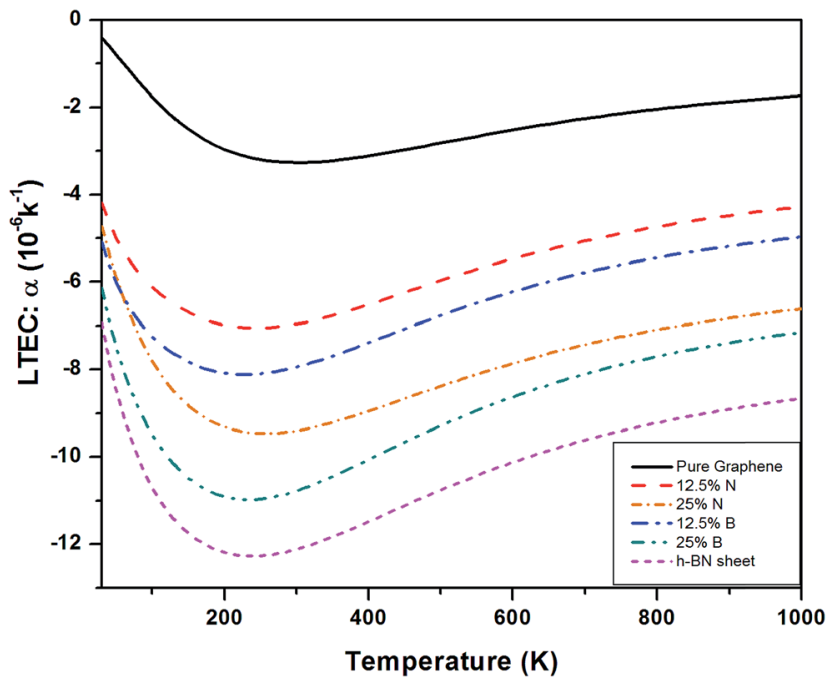

Fig. 11 Temperature dependence of LTEC for pure graphene compared with $\mathrm{B}$ and $\mathrm{N}$ doped graphene and single layer $\mathrm{h}-\mathrm{BN}$ sheet.

74.6 $\mathrm{N} \mathrm{m}^{-1}$ for $\mathrm{h}-\mathrm{BN}$ sheet which is much smaller than surface bulk modulus of pure graphene.

3.3.3 Grüneisen parameter. The mode dependent Grüneisen parameter for h-BN sheet has been obtained for various phonon branches corresponding to different q-values and shown in Fig. 9(a). The Grüneisen parameter corresponding to lowest transverse acoustic mode is negative in the whole region with lowest values approaching as low as - 230 larger than doped and pure graphene case as shown in Fig. 9(b). There is a gap between ZA mode Grüneisen parameters and other acoustic and optical mode Grüneisen parameters contrary to the case of graphene as shown in Fig. 2 where there is no gap. This observation is similar to the observation of difference in phonon dispersion curve of graphene and h-BN.

3.3.4 Thermal expansion coefficient. For calculating LTEC of single layer h-BN sheet, several authors have used various methods ${ }^{\mathbf{1 3 , 2 4 , 4 2 - 4 4}}$ using various experimental techniques ${ }^{\mathbf{4 2 - 4 4}}$ and theoretical models. ${ }^{13,24}$ Sevik $^{13}$ theoretically estimated the LTEC in the temperature range of $0-1500 \mathrm{~K}$ by doing calculations using DFPT as implemented in VASP software. Thomas et al. ${ }^{24}$ have investigated LTEC in a huge range of temperature using molecular dynamics. The results of various experimental techniques for bulk h-BN crystals ${ }^{\mathbf{4 0 , 4 1}}$ are in the temperature range 0$400 \mathrm{~K}$ and lower than the theoretical single sheet estimates given by Sevik. ${ }^{13}$

We obtained the behavior of LTEC for h-BN sheet using DFPT under QHA as shown in Fig. 10(a). Although the RT value of LTEC is much larger than that determined by Sevik ${ }^{\mathbf{1 3}}$ of the order of $-12.86 \times 10^{-6}$ but the behavior is in conformity with $\mathrm{B}$ and $\mathrm{N}$ doped structures. The negative thermal expansion can be explained by the existence of more negative Grüneisen parameters corresponding to the transverse acoustic (ZA) mode and lower surface bulk modulus compared to graphene explains the occurrence of negative thermal expansion. The experimentally measured values in the temperature range $0-400 \mathrm{~K}$ are for bulk h-BN crystals which are lower than estimates for single h-BN sheet. The prominent difference in estimates arises due to the strong anharmonic character of h-BN structure, which is not taken into account in QHA calculations. Alternately, the LTEC values predicted by Thomas et al. ${ }^{24}$ based on fully atomistic simulations gives a lower estimate compared with Sevik. ${ }^{13}$ Fig. 10(b) shows branch dependent LTEC which depicts the contribution of ZA branch is of utmost importance to total thermal expansion as compared to other branches.

Fig. 11 shows a comparison of LTEC for pure and doped graphene compared with h-BN sheet. The graph trend shows an increase in negative thermal expansion with increase in $\mathrm{N}$ and $\mathrm{B}$ doping and further increases in h-BN sheet. We also observe that B doping makes the LTEC value more negative as compared to same doping percentage of $\mathrm{N}$ atom. Further we notice that our estimates are overestimating the LTEC values for h-BN sheet when compared to ref. 13 by a factor close to 2 . However, our study of systematic doping by increasing $\mathrm{B}$ and $\mathrm{N}$ concentrations follows the pattern of LTEC as shown in Fig. 11 quite well where we present results for doping of graphene with individual $\mathrm{B}$ and $\mathrm{N}$ atoms going from $12.5 \%$ to $25 \%$ and finally $50 \% \mathrm{~B}$ and $\mathrm{N}$ doping together creating h-BN sheet.

\section{Summary and conclusions}

In this paper, we have made a detailed first-principles study using the quasi-harmonic approximation at the GGA-PBE level to obtain the finite temperature dependence of LTEC in pure graphene. The thermal expansion coefficient is negative in the whole temperature region under study and is in qualitatively good agreement with available theoretical data although it deviates from experimental studies especially in the low temperature region. In the high temperature region our results are in better agreement with experiments as compared to some other calculations.

Thereafter we have extended the calculation to doped graphene. We have already shown that boron and nitrogen doped graphene is an interesting material whose band gap and other electronic properties can be designed by varying the concentration and type of the dopant. ${ }^{\mathbf{6}, 8}$ The calculation of thermal properties is thus of great importance for using these doped materials in practical applications.

We notice that there is consistent trend of increase in negative Grüneisen parameters with $\mathrm{B}$ and $\mathrm{N}$ doping which continues even for h-BN sheet. The values of Grüneisen parameters tend to vary by a factor of 5 or more for $\mathrm{B}$ or $\mathrm{N}$ doping upto $25 \%$. The same concentration of B atom doping brings about larger change in the negative LTEC value as compared to $\mathrm{N}$ doping. The results for thermal expansion presented here for doped graphene sheet are new.

We extend this approach further by substituting all the carbon atoms by $\mathrm{B}$ and $\mathrm{N}$ atoms to obtain h-BN sheets. We have presented a study of h-BN phonon dispersion and estimated its linear thermal expansion coefficient similarly. The phonon frequencies and phonon dispersions are in good agreement with various theoretical and experimental results. Some calculations report results for thermal expansion of h-BN sheets. Although our results follow similar qualitative temperature 
dependence, the results differ by about a factor of two. Our results (Fig. 11) follow a pattern showing a systematic increase in thermal expansion values as the concentration of dopants increases.

On the basis of the results acquired here for various pure and different doping concentrations of 2D graphene it emerges that the negative thermal expansion originates from ZA modes. These modes result in highly negative Grüneisen parameters and their contribution dominates from the other planar modes. The planar modes (LA, LO, TA, TO) and the remaining transverse modes ( $\mathrm{ZO}$ ) contribute positively but their combined effect remains systematically smaller than the negative contribution from ZA mode. It seems that this feature will always be a unique feature of all 2D materials. Exceptions could be those 2D materials like $\mathrm{MoS}_{2}$ and $\mathrm{MoSe}_{2}$ having larger number of atoms in unit cell and many more branches in planner directions contributing positively to thermal expansion which may end up in combining to nullify most of the negative contribution from ZA mode as measured by Sevik. ${ }^{13}$

Experimental measurements for thermal expansion for graphene lack consistency. There is significant variation and unusual temperature dependence in low temperature region. More experimental work for pure graphene as well as for doped graphene for thermal expansion would be useful to correlate theoretical results.

\section{Acknowledgements}

We are grateful to VASP and phonopy team for providing the code, the departmental computing facilities at Department of Physics, Panjab University, Chandigarh and the HPC facilities at IUAC (New Delhi). VKJ acknowledges great support from Alexander von Humboldt for support as a Guest Professor at University of Würzburg.

\section{References}

1 K. S. Novoselov, A. K. Geim, S. V. Morozov, D. Jiang, et al., Electric field in atomically thin carbon films, Science, 2004, 306(5696), 666-669.

2 A. K. Geim and K. S. Novoselov, The rise of graphene, Nat. Mater., 2007, 6(3), 183-191.

3 A. K. Geim, Graphene: status and prospects, Science, 2009, 324(5934), 1530-1534.

4 A. H. Castro Neto, F. Guinea, N. M. R. Peres, K. S. Novoselov and A. K. Geim, The electronic properties of graphene, Rev. Mod. Phys., 2009, 81, 109-162.

5 P. Avouris, Z. Chen and V. Perebeinos, Carbon based electronics, Nat. Nanotechnol., 2007, 2(10), 605-615.

6 P. Rani and V. K. Jindal, Designing band gap of graphene by B and N dopants, RSC Adv., 2013, 3(3), 802-812.

7 P. Rani and V. K. Jindal, Stability and electronic properties of isomers of b/n co-doped graphene, Appl. Nanosci., 2014, 4(8), 989-996.

8 P. Rani, G. S. Dubey and V. K. Jindal, DFT study of optical properties of pure and doped graphene, Phys. E, 2014, 62, 28-35.
9 D. L. Nika, E. P. Pokatilov, A. S. Askerov and A. A. Balandin, Phonon thermal conduction in graphene: Role of Umklapp and edge roughness scattering, Phys. Rev. B, 2009, 79(15), 155413.

10 N. Mounet and N. Marzari, First-principles determination of the structural, vibrational and thermodynamic properties of diamond, graphite, and derivatives, Phys. Rev. B: Condens. Matter Mater. Phys., 2005, 71(20), 205214.

11 K. V. Zakharchenko, M. I. Katsnelson and A. Fasolino, Finite temperature lattice properties of graphene beyond the quasiharmonic approximation, Phys. Rev. Lett., 2009, 102, 046808.

12 J. W. Jiang, J. S. Wang and B. Li, Young's modulus of graphene: A molecular dynamics study, Phys. Rev. B: Condens. Matter Mater. Phys., 2009, 80, 205429.

13 C. Sevik, Assessment on lattice thermal properties of twodimensional honeycomb structures: Graphene, h-BN, h$\mathrm{MoS}_{2}$, and h-MoSe 2 , Phys. Rev. B: Condens. Matter Mater. Phys., 2014, 89, 035422.

14 C. W. Kim, S. H. Kang and Y. K. Kwon, Rigid unit modes in $\mathrm{sp}-\mathrm{sp}^{2}$ hybridized carbon systems: Origin of negative thermal expansion, Phys. Rev. B: Condens. Matter Mater. Phys., 2015, 92, 245434.

15 J. W. Jiang, B. S. Wang, J. S. Wang and H. S. Park, A review on flexural mode of graphene: lattice dynamics, thermal conduction, thermal expansion, elasticity, and nanomechanical resonance, J. Phys.: Condens. Matter, 2015, $27(8), 083001$.

16 K. H. Michel, S. Costamagna and F. M. Peeters, Theory of thermal expansion in 2D crystals, Phys. Status Solidi B, 2015, 252(11), 2433-2437.

17 K. H. Michel, S. Costamagna and F. M. Peeters, Theory of anharmonic phonons in two-dimensional crystals, Phys. Rev. B: Condens. Matter Mater. Phys., 2015, 91(13), 134302.

18 W. Bao, F. Miao, Z. Chen, et al., Controlled ripple texturing of suspended graphene and ultrathin graphite membranes, Nat. Nanotechnol., 2009, 4, 562.

19 D. Yoon, Y. W. Son and H. Cheong, Negative Thermal Expansion Coefficient of Graphene Measured by Raman Spectroscopy, Nano Lett., 2011, 11(8), 3227-3231.

20 W. Pan, J. Xiao, J. Zhu, C. Yu, et al., Biaxial Compressive Strain Engineering in Graphene/Boron Nitride Heterostructures, Sci. Rep., 2012, 2, 893.

21 X. Blase, A. Rubio, S. G. Louie and M. L. Cohen, Quasiparticle band structure of bulk hexagonal boron nitride and related systems, Phys. Rev. B: Condens. Matter Mater. Phys., 1995, 51, 6868.

22 K. Watanabe, T. Taniguchi and H. Kanda, Direct-bandgap properties and evidence for ultraviolet lasing of hexagonal boron nitride single crystal, Nat. Mater., 2004, 3, 404-409.

23 D. Golberg, Y. Bando, Y. Huang, T. Terao, M. Mitome, C. Tang and C. Zhi, Boron nitride nanotubes and nanosheets, ACS Nano, 2010, 4(6), 2979-2993.

24 S. Thomas, K. M. Ajith, S. Chandra and M. C. Valsakumar, Temperature dependent structural properties and bending rigidity of pristine and defective hexagonal boron nitride, J. Phys.: Condens. Matter, 2015, $27(31), 315302$. 
$25 \mathrm{Y}$. Hu, J. Chen and B. Wang, On the intrinsic ripples and negative thermal expansion of graphene, Carbon, 2015, 95, 239-249.

26 S. Mann, P. Rani, R. Kumar, G. S. Dubey and V. K. Jindal, Thermodynamic properties of pure and doped $(\mathrm{B}, \mathrm{N})$ graphene, $R S C$ Adv. , 2016, 6(15), 12158-12168.

27 G. Kresse and J. Furthmüller, Efficient iterative schemes for $a b$ initio total-energy calculations using a plane-wave basis set, Phys. Rev. B: Condens. Matter Mater. Phys., 1996, 54(16), 11169.

28 G. Kresse and D. Joubert, From ultrasoft pseudopotentials to the projector augmented-wave method, Phys. Rev. B: Condens. Matter Mater. Phys., 1999, 59(3), 1758.

29 A. Togo, F. Oba and I. Tanaka, First-principles calculations of the ferroelastic transition between rutile-type and $\mathrm{CaCl}_{2}$ type $\mathrm{SiO}_{2}$ at high pressures, Phys. Rev. B: Condens. Matter Mater. Phys., 2008, 78(13), 134106.

30 V. K. Jindal and J. Kalus, Calculation of thermal expansion and phonon frequency shift in deuterated naphthalene, Phys. Status Solidi B, 1986, 133, 189.

31 R. Bhandari and V. K. Jindal, Calculation of thermal expansion and implicit phonon frequency shift in deuterated anthracene, J. Phys.: Condens. Matter, 1991, 3, 899-907.

32 J. P. Perdew, K. Burke and M. Ernzerhof, Generalized Gradient Approximation Made Simple, Phys. Rev. Lett., 1996, 77(18), 3865-3868.

33 P. E. Blöchl, Projector augmented-wave method, Phys. Rev. B: Condens. Matter Mater. Phys., 1994, 50(24), 17953.

34 S. Reich and C. Thomsen, Elastic properties of carbon nanotubes under hydrostatic pressure, Phys. Rev. B: Condens. Matter Mater. Phys., 2002, 65, 153407.

35 G. Kalosakas, N. N. Lathiotakis, C. Galiotis and K. Papagelis, In-plane force fields and elastic properties of graphene, $J$. Appl. Phys., 2013, 113, 134307.
36 K. V. Zakharchenko, M. I. Katsnelson and A. Fasolino, Finite temperature lattice properties of graphene beyond the quasiharmonic approximation, Phys. Rev. Lett., 2009, 102(4), 046808.

37 K. Z. Milowska, M. Woińska and M. Wierzbowska, Contrasting Elastic Properties of Heavily B-and N-doped Graphene with Random Impurity Distributions Including Aggregates, J. Phys. Chem. C, 2013, 117(39), 20229-20235.

$38 \mathrm{~L}$. Wirtz and A. Rubio, $A b$ initio calculations of the lattice dynamics of boron nitride nanotubes, Phys. Rev. B: Condens. Matter Mater. Phys., 2003, 68(4), 045425.

39 D. S. Portal and E. Hernandez, Vibrational properties of single-wall nanotubes and monolayers of hexagonal $\mathrm{BN}$, Phys. Rev. B: Condens. Matter Mater. Phys., 2002, 66(23), 235415.

40 J. Serrano, A. Bosak, R. Arenal, M. Krisch, K. Watanabe, T. Taniguchi, et al., Vibrational Properties of Hexagonal Boron Nitride: Inelastic X-Ray Scattering and $A b$ Initio Calculations, Phys. Rev. Lett., 2007, 98, 095503.

41 S. Reich, A. C. Ferrari, R. Arenal, A. Loiseau, I. Bello and J. Robertson, Resonant Raman scattering in cubic and hexagonal boron nitride, Phys. Rev. B: Condens. Matter Mater. Phys., 2005, 71, 205201.

42 G. L. Belenkii, E. Y. Salaev, R. A. Suleimanov, N. A. Abdullaev and V. Shteinshraiber, The nature of negative linear expansion in layer crystals $\mathrm{C}, \mathrm{Bn}, \mathrm{GaS}, \mathrm{GaSe}$ and InSe, Solid State Commun., 1985, 53(11), 967-971.

43 W. Paszkowicz, J. Pelka, M. Knapp, T. Szyszko and S. Podsiadlo, Lattice parameters and anisotropic thermal expansion of hexagonal boron nitride in the $10-297.5 \mathrm{~K}$ temperature range, Appl. Phys. A: Mater. Sci. Process., 2002, 75(3), 431-435.

44 B. Yates, M. Overy and O. Pirgon, The anisotropic thermal expansion of boron nitride, Philos. Mag., 1975, 32(4), 847857. 